\title{
A rare case of multiple bronchial artery aneurysms associated with a double aortic arch
}

\author{
Rameysh Danovani Mahmood ${ }^{1}$, MB BCh BAO, FRCR, Zhi Yong $\underline{C h e n}^{2}$, MBBS, MRCP, Teck Boon $\underline{L o w}^{2}$, MB BCh BAO, MRCP,
} Keng Sin $\mathrm{Ng}^{1}$, MBBS, FRCR

\begin{abstract}
Bronchial artery aneurysm is uncommon, and the occurrence of multiple aneurysms arising from a bronchial artery is even rarer. To date, there has been only one published case report describing double bronchial artery aneurysms. We herein describe a case of three aneurysms arising from a left bronchial artery, accompanied by multiple bilateral hypertrophied bronchial and intercostobronchial arteries, as well as a double aortic arch. Bronchial artery aneurysm is potentially life-threatening, and immediate treatment is recommended to minimise the potential risk of rupture. The aneurysms in our case were successfully treated via transcatheter arterial embolisation using coils.
\end{abstract}

Keywords: aneurysm, bronchial artery, embolisation, hypertrophy, multiple aneurysms

\section{INTRODUCTION}

Although bronchial artery aneurysm (BAA) accounts for less than $1 \%$ of cases of selective bronchial arteriography, this rare condition is a known cause of haemoptysis and mediastinal haematoma. ${ }^{(1,2)}$ It is potentially life-threatening, and thus, treatment should be initiated as soon as diagnosis is made. No direct aetiology has been established; however, it has been found to be associated with chronic pulmonary diseases and vascular abnormalities. Transcatheter arterial embolisation (TAE) is the treatment of choice, although surgical treatment may be the best option in some cases.

To the best of our knowledge, only one published case report in the English literature has described a case of double BAAs to date. ${ }^{(3)}$ We herein report a case of three aneurysms arising from a left bronchial artery in a patient who presented with haemoptysis. In our case, there was no associated pulmonary abnormality, although the aneurysms were found in association with a double aortic arch, as well as hypertrophied and tortuous bronchial and intercostobronchial arteries. The aneurysms were successfully treated via TAE using embolisation coils.

\section{CASE REPORT}

A 73-year-old man with a background history of hypertension, hyperlipidaemia and mild aortic stenosis presented to our emergency department with two episodes of haemoptysis, amounting to a teaspoon full. He was a non-smoker and denied having chronic pulmonary diseases, or loss of weight or appetite. He was haemodynamically stable, but was admitted for further investigations and treatment.

The patient was subsequently referred to the radiology department for computed tomography (CT) thoracic aortography. In addition to the double aortic arch (Fig. 1) and a right-sided descending aorta, several hypertrophied bronchial arteries (Fig. 2), intercostobronchial arteries and thyrocervical trunks were also seen bilaterally. One of the left hypertrophied bronchial arteries was associated with three saccular aneurysms (Fig. 2), the largest of which measured approximately $2.0 \mathrm{~cm} \times 1.5 \mathrm{~cm}$. One of the aneurysms was partially thrombosed, with mural calcifications. There was no evidence of bronchiectasis on CT.

The findings were confirmed the following day by selective bronchial arteriography (Fig. 3), performed via a right common femoral artery puncture. The hypertrophied right and left bronchial arteries were selected using a $5 \mathrm{Fr} \mathrm{CHG}$ 2.5 catheter $(\operatorname{Cook} \AA$, Cook Medical Inc, IN, USA), and were later superselected using a $2.7 \mathrm{Fr}$ microcatheter (Progreat $^{\mathrm{TM}}$, Terumo Medical Corporation, NJ, USA). The microcatheter was advanced beyond the aneurysms to enable distal embolisation using polyvinyl alcohol (PVA) particles of 355-500 $\mu \mathrm{m}$ in size (Contour ${ }^{\circledR}$, Boston Scientific, MA, USA) for treatment of haemoptysis. After achieving satisfactory stasis, the arterial branches just beyond the aneurysms were coiled to prevent possible retrograde filling. The three aneurysm sacs were later coiled extensively by using multiple complex helical as well as fibered platinum coils of size 4-7 mm (Vortex 18, Boston Scientific, MA, USA; Nester ${ }^{\circledR}$, Cook Medical Inc, IN, USA) until satisfactory non-filling was achieved (Fig. 4). An Amplatzer vascular plug was considered for the remnant inflow bronchial artery proximal to the aneurysm, pending the findings of postembolisation CT angiography. The rest of the hypertrophied bilateral bronchial, intercostobronchial and thyrocervical arteries were also superselected using the microcatheter and embolised using PVA particles in the same setting. There was no hypertrophied bronchial artery arising from the internal mammary arteries. Post-procedure follow-up CT showed no opacification of the origin of the bronchial artery supplying the coiled aneurysms, and the BAAs were completely occluded. No further intervention was performed and the Amplatzer vascular plug was not inserted.

Post embolisation, the patient developed a low-grade fever for five days. Blood tests showed normal white blood cell

${ }^{1}$ Department of Radiology, ${ }^{2}$ Department of Respiratory Medicine, Changi General Hospital, Singapore

Correspondence: Dr Rameysh Danovani Mahmood, Registrar, Department of Radiology, Changi General Hospital, 2 Simei Street 3, Singapore 529889.

rameysh_danovani_mahmood@cgh.com.sg 

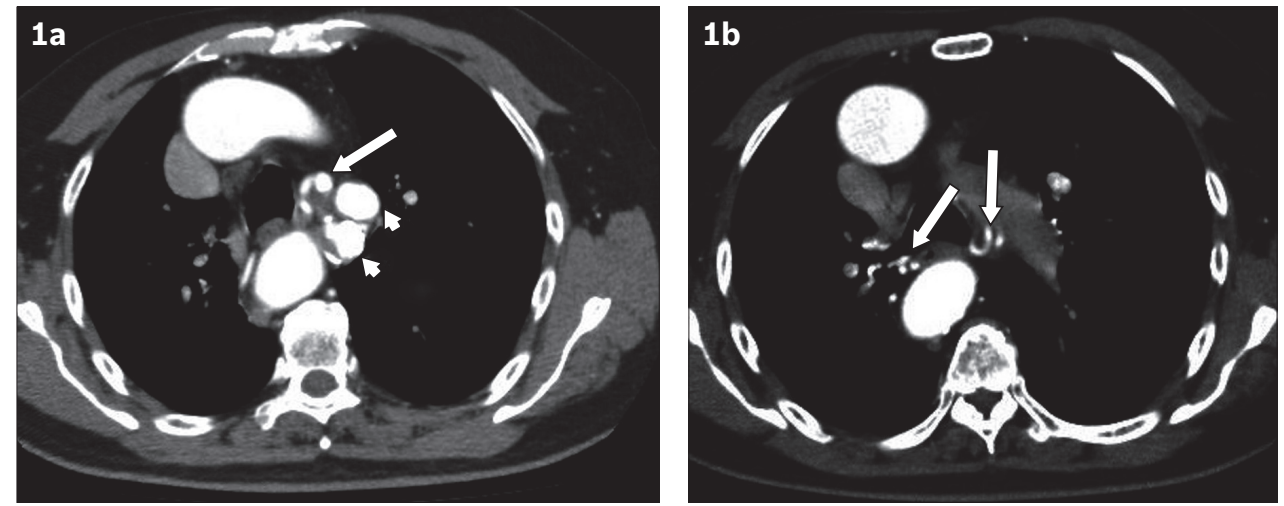

Fig. 1 Axial CT images in the arterial phase performed at initial presentation show (a) the hypertrophied bronchial artery (arrow) and two of the three aneurysms (arrowheads), and (b) the presence of bilaterally hypertrophied and tortuous bronchial arteries at a slightly more inferior level (arrows).

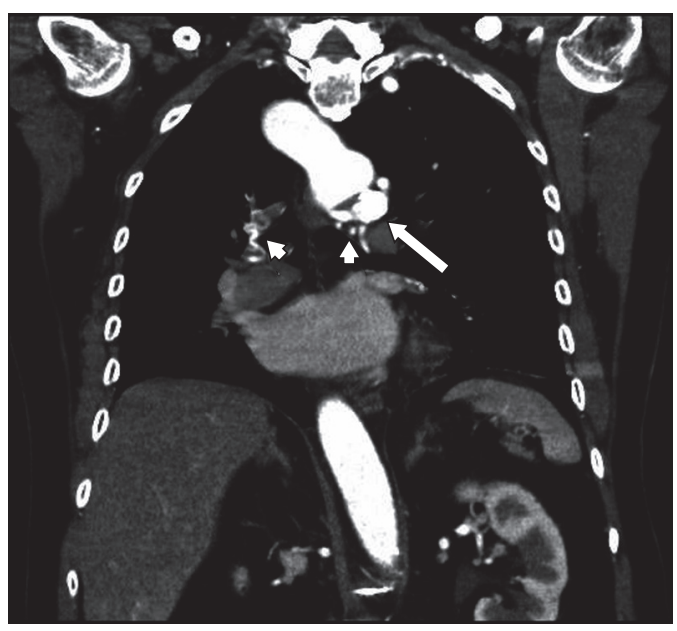

Fig. 2 Coronal reconstruction from CT thoracic aortogram shows aneurysms of the bronchial artery (arrow), accompanied by bilateral hypertrophied bronchial arteries (arrowheads). The right-sided portion of the double aortic arch is also shown.

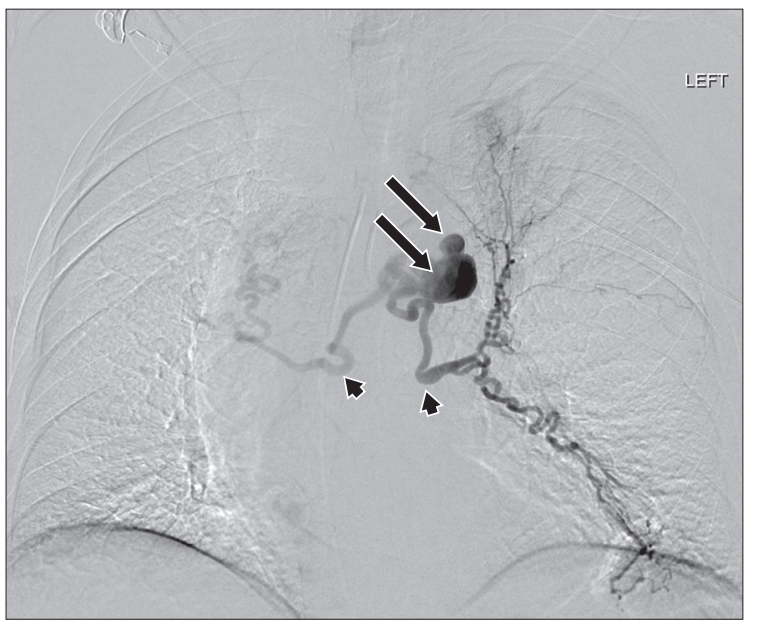

Fig. 3 Digital subtraction angiogram from selective bronchial arteriography shows the hypertrophied bronchial arteries (arrowheads), which are associated with the saccular aneurysms (arrows).

counts and inflammatory markers with no identifiable source of infection. The patient was treated conservatively, as the fever was attributed to post-embolisation syndrome. In view of the presence of concomitant congenital vascular anomalies (i.e. double aortic arch and hypertrophied bronchial arteries), the patient underwent further biochemical workup for possible connective tissue disease, which revealed normal results.

The patient had an uneventful recovery and was discharged well after ten days of hospitalisation. On follow-up at the clinic eight months after embolisation, he was well, with no recurrence of haemoptysis or hospital admission in the interim. Chest radiography performed during this visit showed no evidence of migration of coils, and no CT was performed. At the time of this writing, he was awaiting a subsequent clinical and chest radiography follow-up at 12 months.

\section{DISCUSSION}

In the current literature, the uncommon occurrence of BAA has been well documented, with most cases describing a single aneurysm developing in association with chronic inflammatory pulmonary diseases such as bronchiectasis and tuberculosis. Although the exact pathophysiology is unknown, this association may in part be related to hyperaemia in a diseased lung, resulting in the recruitment of bronchial arteries.

To date, the only published report of a case of double BAAs described its occurrence in association with pulmonary bronchiectasis. ${ }^{(3)}$ In contrast, our patient did not show evidence of bronchiectasis, active disease or stigmata of prior pulmonary tuberculosis, or other chronic pulmonary diseases. Repeated blood cultures and sputum acid-fast bacilli smears and cultures were negative. We believe that our case report is the first to describe three aneurysms arising from a bronchial artery, with no apparent association with chronic lung disease.

In addition, the occurrence of BAAs in our case was associated with a double aortic arch, as well as hypertrophied and tortuous bronchial arteries. This could potentially represent a manifestation of an underlying systemic connective tissue disease. However, there was an absence of positive family history and clinical features. BAAs have also been reported to be associated with autoimmune vasculitis such as polyarteritis nodosa and HughesStovin syndrome, as well as hyperimmunoglobulinaemia E syndrome. ${ }^{(4-6)}$ However, test results for a series of autoimmune markers (such as antinuclear antibody) in our case were negative. Furthermore, there was an absence of multi-organ involvement and progressive clinical course. There was also a lack of evidence 

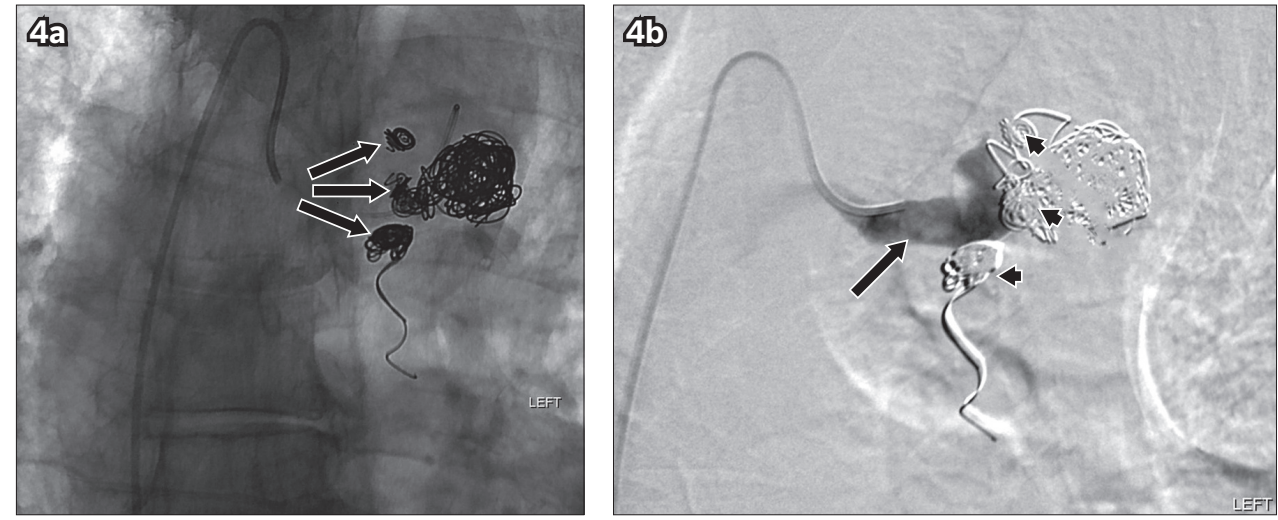

Fig. 4 Post-embolisation angiograms show (a) the extensively coiled aneurysms (arrows), and (b) complete occlusion with non-opacification of the aneurysms and their outflow vessels (arrowheads), and opacification of a short segment of the inflow portion of the bronchial artery at the end of embolisation (arrow), which was not evident in the follow-up CT (not shown).

of sepsis to suggest inflammatory conditions such as infective vasculitis.

Other vascular abnormalities that have been implicated in BAA include Behcet's disease. ${ }^{(7)}$ BAA has also been reported to be associated with cystic fibrosis and other congenital pulmonary diseases such as pulmonary agenesis and sequestration. ${ }^{(1,3)}$ Trauma is also a recognised cause of BAA. ${ }^{(1)}$ Our elderly patient did not have a prior history of any of these conditions or precipitating trauma. The aetiology of vascular ectasia and BAA in our patient therefore remains unknown.

Depending on its location, BAA may present in several ways. Haemoptysis is common in an intrapulmonary BAA, while mediastinal BAA may present with complications such as rupture and mediastinal haematoma, which cause acute thoracic pain and may mimic conditions such as aortic dissection. Our patient presented with only haemoptysis. Chronic cases of mediastinal BAA may present with symptoms in relation to mass effect.

$\mathrm{CT}$, which is frequently used to investigate the cause of haemoptysis, should be performed in the arterial phase. Interventional radiologists may find it useful to conduct a CT study prior to conventional angiography. CT also provides good evaluation of the lungs and other mediastinal structures. It has been accepted that BAA should be treated as soon as the diagnosis is made, regardless of symptoms, in order to minimise the risk of life-threatening complications such as rupture. ${ }^{(8)}$ The aneurysm diameter alone does not seem to be an independent risk factor. ${ }^{(8)}$ In our case, the patient underwent endovascular intervention the following day after diagnosis was made.

Endovascular procedures have become the treatment of choice for BAA, as they are less invasive than surgical extirpation and able to provide successful exclusion of the aneurysm, with a shorter hospital stay. ${ }^{(3)}$ As in our case, TAE is the endovascular procedure commonly employed with the aim to totally occlude the aneurysm.

As our patient presented with haemoptysis, we opine that it was ideal to first embolise the arteries distally with PVA particles, to ensure the symptom was controlled before occlusion of the feeding artery and aneurysms was performed. The use of PVA particles for embolisation of bronchial arteries is common and has previously been reported by Yoon et al and Kalva. ${ }^{(9,10)}$
Following this, we proceeded to coil the efferent vessels to avoid retrograde filling of the aneurysms, which could potentially cause enlargement or rupture. Finally, all three aneurysms were carefully packed with coils. The use of coils for embolisation of aneurysm sacs has frequently been reported in the literature. ${ }^{(1,3)}$

Successful embolisation using liquid embolics such as $\mathrm{N}$-butyl-2-cyanoacrylate (NBCA) has also been reported. ${ }^{(11)}$ Aburano et al described successful embolisation of a BAA with fistulous communication between the aneurysm sac and pulmonary artery, using NBCA. ${ }^{(11)}$ The suitability of each embolic material must be carefully considered on a case-by-case basis, as some may be predisposed to technical disadvantages, as well as potential complications. NBCA, for example, carries with it the risks of premature catheter occlusion and tissue necrosis.

Transcatheter stenting across the bronchial artery and aneurysms is considered a potential means of treatment for aneurysms. However, this procedure was deemed unsuitable and thus not performed in our case, mainly due to the anticipated technical difficulty, given the tortuosity of the involved vessels and the presence of multiple aneurysms, which may render occlusion ineffective. Furthermore, there have been no published reports on the efficacy of this technique. However, there are a few documented cases of endovascular aortic stenting across the orifices of the feeding vessels. ${ }^{(1,3,12,13)}$ Nevertheless, this procedure was not considered for our patient, as there was adequate length of the inflow segment to the aneurysm to provide safe and effective distal occlusion.

As surgical extirpation is more invasive than endovascular procedures, it has only been described in cases of severe complications such as rupture, ${ }^{(2)}$ or in cases where TAE is not feasible or has failed. Regardless of the procedure of choice, post-procedure follow-up imaging is certainly required, to ensure that reperfusion of the aneurysm sac can be identified and treated early.

In summary, we described a very rare and unusual case of multiple BAAs arising from a bronchial artery, which were successfully treated with embolisation coils and PVA particles. The aneurysms were associated with a double aortic arch, as well as hypertrophied and tortuous bronchial arteries; such an 
occurrence has not been previously reported. However, the exact aetiology and pathophysiology of the aneurysms in our case remain unknown.

\section{REFERENCES}

1. Kasashima F, Endo M, Kosugi I, et al. Mediastinal bronchial artery aneurysm treated with a stent-graft. J Endovasc Ther 2003; 10:381-5.

2. Kalangos A, Khatchatourian G, Panos A, Faidutti B. Ruptured mediastina bronchial artery aneurysm: a dilemma of diagnosis and therapeutic approach. J Thorac Cardiovasc Surg 1997; 114:853-6.

3. Tsolaki E, Salviato E, Coen M, Galeotti R, Mascoli F. Double right bronchia artery aneurysm treated with combined procedures. Eur J Vasc Endovasc Surg 2007; 34:537-9.

4. Lee YJ, Park SS, Kim SY, et al. A case of systemic polyarteritis nodosa involving bronchial artery. Sarcoidosis Vasc Diffuse Lung Dis 2010; 27:164-8.

5. Khalid U, Saleem T. Hughes-Stovin syndrome. Orphanet J Rare Dis 2011; $6: 15$.
6. Connolly B, Manson D, Khattak S, Burrows P. Bronchial artery aneurysm in hyperimmunoglobulinemia E syndrome. Pediatr Radiol 1994; 24:592-3.

7. Cil BE, Geyik S, Akmangit I, et al. Embolization of a giant pulmonary artery aneurysm from Behcet disease with use of cyanoacrylate and the "bubble technique". J Vasc Interv Radiol 2005; 16:1545-9.

8. Takahashi Y, Tsutsumi Y, Monta O, et al. Stent grafting for giant bronchial artery aneurysm disguised as an aneurysm with multiple feeding arteries. Ann Thorac Surg 2010; 89:1652-4.

9. Yoon W, Kim JK, Kim YH, Chung TW, Kang HK. Bronchial and nonbronchial systemic artery embolization for life-threatening hemoptysis: a comprehensive review. Radiographics 2002; 22:1395-409.

10. Kalva SP. Bronchial artery embolization. Tech Vasc Interv Radiol 2009; 12:130-8.

11. Aburano $H$, Kawamori $Y$, Horiti $Y$, et al. Bronchial artery aneurysm embolization with NBCA. Cardiovasc Intervent Radiol 2006; 29:1141-3.

12. Sakai T, Razavi MK, Semba CP, et al. Percutaneous treatment of bronchial artery aneurysm with use of transcatheter coil embolization and thoracic aortic stent-graft placement. J Vasc Interv Radiol 1998; 9:1025-8.

13. Sanchez E, Alados P, Zurera L, et al. Bronchial artery aneurysm treated with aortic stent graft and fibrin sealant. Ann Thorac Surg 2007; 83:693-5. 